\title{
Sentido de Coherencia asociado con los Eventos Estresantes de la Vida en adultos mayores
}

\author{
Sense of Coherence associated with \\ Life Stressful Events in elderly
}

\section{Senso de coerência associado a eventos Estressantes da Vida em Idosos}

\author{
C.A. Salazar-Moreno ${ }^{\mathrm{a}}$, M.M. Alonso-Castillo ${ }^{\mathrm{b}^{*}}$, \\ F.R. Guzmán-Facundoc, K.S. López-García ${ }^{\mathrm{d}}$ \\ ORCID \\ a $0000-0001-7130-2274$ \\ c0000-0002-6951-8989 \\ b $0000-0002-7197-8116$ \\ d $\underline{0000-0002-9462-7140}$
}

Universidad Autónoma de Nuevo León, Facultad de Enfermería, Nuevo León, México

Recibido: 4 marzo 2020

Aceptado: 25 junio 2020

\section{RESUMEN}

Introducción: Los Adultos Mayores (AM) se consideran una población vulnerable debido a la experiencia constante de Eventos Estresantes de la Vida (EEV), es decir, acontecimientos que generan angustia, preocupación y mortificación en el AM, los cuales al no ser resueltos pueden derivar en una problemática de salud de mayor complejidad. La respuesta a este fenómeno puede estar en el Modelo Salutogénico y su constructo principal el Sentido de Coherencia (SOC por sus siglas en inglés), el cual permite al individuo hacer frente al estrés y alcanzar la salud; sin embargo, aún no hay un consenso respecto al efecto del SOC sobre los EEV en los AM.

Objetivo: Conocer la relación entre los EEV y el SOC en AM del norte de México.

*Autora para correspondencia. Correo electrónico: magdalena_alonso@hotmail.com https://doi.org/10.22201/eneo.23958421e.2020.3.853

1665-7063/@ 2020 Universidad Nacional Autónoma de México, Escuela Nacional de Enfermería y Obstetricia. Este es un artículo Open Access bajo la licencia CC BY-NC-ND (http://creativecommons.org/licenses/by-nc-nd/4.o/). 
Método: Se realizó un estudio descriptivo correlacional, con un muestreo probabilístico en 108 AM. Los instrumentos utilizados fueron el Inventario de Estrés en la Vida de Adultos Mayores y el Cuestionario de Orientación Hacia la Vida (SOC-13).

Resultados: $77.8 \%$ pertenece al sexo femenino, los eventos más experimentados en los últimos doce meses fueron el deterioro de la memoria (54.6\%) y la presencia de una lesión o enfermedad (50\%). El Modelo de Regresión Simple reveló que el SOC fue predictor negativo de los EEV $(\beta=-.652, p=.001)$.

Conclusión: El SOC podría ayudar al AM a comprender, manejar y dar significado a los EEV que experimenta y de esta forma percibir su entorno como menos amenazante.

Palabras clave: Envejecimiento; estrés psicológico; anciano; sentido de coherencia; México.

\section{ABSTRACT}

Introduction: Older adults are considered a vulnerable population because of their constant exposure to Life Stressful Events, including those which provoke anguish, concern, and distress, and which, if not adequately addressed, could even become a more complex health problem. The answer to this situation could be the Salutogenic Model and its main construct-Sense of Coherence-which supports the individual to face stress and reach a state of health. Nevertheless, a consensus has not yet been achieved regarding the effects of using this model on older adults suffering from Life Stressful Events.

Objective: To better understand the relationship between Life Stressful Events and the construct Sense of Coherence among older adults.

Method: This is a descriptive and correlational study with probabilistic sampling and 108 older adults. The instruments used were a Stress in the Life of Older Adults Inventory, and a Questionnaire on Orientation Towards Life (Sense of Coherence - 13).

Results: $77.8 \%$ participants were female. The most frequently experienced stressful events during the last 12 months were: memory deterioration (54.6\%) and anguish due to lesions of illnesses (50\%). A Simple Regression Model revealed that Sense of Coherence was a negative predictor of Life Stressful Events ( $\beta=-.652, p=.001$ ).

Conclusion: Using the construct Sense of Coherence can help older adults understand and manage their Life Stressful Events so that they can perceive their environment as less threatening.

Keywords: Aging; stress, psychological; aged; sense of coherence; Mexico.

\section{RESUMO}

Introdução: Os Idosos são considerados uma população vulnerável devido a sua experiência constante de Eventos Estressantes da Vida (EEV), quer dizer, eventos que geram angústia, preocupação e mortificação no idosos, os quais quando não resolvidos podem derivar em um problema de saúde mais complexo. A resposta a este fenómeno pode estar no Modelo Salutogénico e seu construto principal o Senso de Coerência (SOC por suas siglas em inglês), que permite ao indivíduo enfrentar o estresse e alcançar a saúde; porém, ainda não há um consenso ao respeito do efeito do SOC sobre os EEV nos idosos.

Objetivo: Conhecer a relação entre os EEV e o SOC em idosos do norte do México. 
Método: Foi realizado um estudo descritivo correlacional, com uma amostragem probabilística em 108 idosos. Os instrumentos utilizados foram o Inventario de Estresse na Vida de Idosos e o Questionário de Orientação Para a Vida (SOC-13).

Resultados: $77.8 \%$ pertence ao sexo feminino, os eventos mais experimentados nos últimos doze meses foram o deterioro da memória (54.6\%) e a presença de uma lesão ou doença (50\%). O Modelo de Regressão Simples revelou que o SOC foi preditor negativo dos EEV ( $\beta=$ $-.652, p=.001)$.

Conclusão: O SOC podia ajudar o idosos a compreender, manejar e dar significado aos EEV que experimenta e desta forma perceber seu entorno como menos ameaçador.

Palavras chave: Envelhecimento; estresse psicológico; idoso; senso de coerência; México.

\section{INTRODUCCIÓN}

Hoy en día la proporción de Adultos Mayores (AM) del planeta aumenta más rápido que cualquier grupo de edad y se espera que para el 2050 se duplique a 22\%. En México residen 15.4 millones de AM, los cuales representan $12.3 \%$ de la población total ${ }^{1-3}$. Los AM en México son considerados un grupo vulnerable debido a que $41 \%$ de ellos viven en situación de pobreza, $11.4 \%$ vive solo, $30 \%$ vive en estado de abandono, solo $39 \%$ permanece económicamente activo, por ello tienen acceso a servicios de salud y solo 16.2\% cuenta con una pensión; además la mitad de la población de AM tiene alguna discapacidad $(49.9 \%)^{3-6}$.

Estos hechos pueden ser considerados como Eventos Estresantes de la Vida (EEV), que son definidos como acontecimientos que generan en el AM angustia, preocupación y mortificación, a su vez, son evaluados como retos o amenazas que requieren de ajustes en la vida; si no son resueltos tienen la posibilidad de derivar en una problemática de salud de mayor complejidad7 ${ }^{7-10}$. El fenómeno de los EEV puede ser abordado a través de la perspectiva salutogénica desarrollada por Aaron Antonovsky, la cual se centra en conocer los orígenes de la salud aun en medio de situaciones de estrés intenso; como resultado de sus estudios surgió el Modelo Salutogénico y su concepto central, el Sentido de Coherencia (SOC) ${ }^{11-15}$.

El SOC fue definido por Antonovsky como una orientación global del mundo y el entorno individual. Expresa hasta qué punto la persona tiene una amplia, resistente y dinámica sensación de confianza que 1) los estímulos que provienen del entorno (interno y externo) en el curso de su vida son estructurados, predecibles y explicables, 2) cuenta con los recursos necesarios para hacerles frente, 3) que estas demandas son desafíos dignos de inversión y compromiso; estas tres dimensiones comprenden el SOC y se denominan comprensibilidad, manejabilidad y significatividad ${ }^{16}$. Los individuos con alto SOC afrontan mejor los EEV y se mantienen más saludables en comparación con aquellos con bajo SOC, que parecen ser más vulnerables y tienen más problemas de salud ${ }^{17-18}$.

Estudios realizados en AM muestran altos niveles de SOC en los participantes ${ }^{14,19,20}$; asimismo otros estudios en AM muestran relaciones negativas entre el SOC y el estrés ${ }^{18,21}$. Además, es importante mencionar que hasta el momento se han identificado escasos estudios en México sobre el SOC en los $\mathrm{AM}^{22-23}$; sin embargo, estos no abordan la relación entre el SOC y los EEV, por lo que no se ha llegado a un consenso sobre el efecto del SOC y los EEV en los AM y se considera que existe un vacío en el conocimiento. 
Aunado a lo anterior, al aumento de la proporción de AM mexicanos y su constante exposición a los EEV, la falta de consenso del efecto del SOC sobre los EEV y los escasos datos producto de la investigación en relación con el SOC y los EEV en AM en México, el objetivo general del presente estudio fue conocer la relación y el efecto del SOC con los EEV en los AM de una región del norte de México.

\section{MATERIAL Y MÉTODOS}

Se realizó un estudio descriptivo correlacional, el cual se llevó a cabo en los meses de julio-octubre del 2019 en 108 AM que asistían a nueve grupos sociales ubicados en los municipios de Santa Catarina, Apodaca y Guadalupe; Nuevo León, México, se utilizó un muestreo probabilístico proporcional al tamaño de cada uno de los nueve estratos. Se incluyeron a aquellos AM que se encontraban ubicados en tiempo, espacio y persona. Para evaluar lo anterior se utilizaron las siguientes preguntas: ¿qué día es hoy?, ¿cómo se llama? y ¿cuánto tiempo lleva viviendo en la colonia? Además, se evaluó la presencia de deterioro cognitivo a través de la versión en español de la Short Portable Mental Status Questionnaire (SPMSO-VE) de Pfeiffer, la cual fue traducida al español por Martínez ${ }^{24}$. Se determinó la exclusión de aquellos que presentaran deterioro cognitivo; sin embargo, este no se observó en ninguno de los participantes.

Se aplicó una Cédula de Datos Personales y dos instrumentos. Para medir el SOC se utilizó el Cuestionario de Orientación Hacia la Vida (SOC-13) versión breve desarrollado por Antonovsky ${ }^{25}$ que tiene como propósito evaluar el SOC como una orientación global para ver el mundo y el entorno individual como comprensible, manejable y significativo, es decir, mide cómo las personas resuelven situaciones o eventos que pueden ser estresantes. Está compuesto por trece preguntas organizadas en tres dimensiones (comprensibilidad, manejabilidad y significatividad). Este cuestionario se deriva del original de 29 reactivos desarrollado por el mismo autor, quien refirió que la versión breve permitiría contestar en un menor tiempo y de este modo reducir la falta de respuestas sin afectar la confiabilidad ni la validez de constructo. El rango de respuesta de las preguntas es de 1 a 7; el puntaje mínimo es de 13 y el máximo es de 91, en el cual a mayor puntaje mayor SOC.

Virués-Ortega, Martínez-Martín, del Barrio y Lozano ${ }^{26}$ realizaron la traducción al español y lo aplicaron en AM de 70 años o más de España obteniendo un Alpha de Cronbach de o.80, Ugarte et al. ${ }^{23}$ lo aplicaron en 265 AM del Norte de México y tuvieron un Alpha de Cronbach de 0.79; en el presente estudio dicho cuestionario obtuvo un Alpha de Cronbach de 0.73.

Para medir la presencia de los EEV se empleó el Inventario de Estrés en la Vida de AM (ELSI, por sus siglas en inglés) elaborado por Aldwin, que tiene como propósito evaluar los EEV que experimenta el AM en los últimos doce meses. Está compuesto por 31 eventos de vida, tiene seis opciones de respuesta que van de o a 5, el puntaje mínimo es de o y el máximo es de 155, el cual indica mayor estrés en la vida.

Alonso ${ }^{27}$ lo aplicó en 112 AM de Monterrey, Nuevo León, México, y realizó el procedimiento de Back Translation con dos traductores profesionales que realizaron la traducción del inglés al español, posteriormente un panel de expertos revisó ambas versiones del español, las cuales dieron como resultado una traducción no solo lingüística sino conceptual. Más adelante, un traductor de lengua inglesa materna tradujo el cuestionario en español nuevamente al inglés y se compararon ambas versiones del cuestionario original y del traducido después del proceso antes señalado ${ }^{28}$. Una vez que se tuvo la versión en español se obtuvo un Alpha de Cronbach de 0.69 y en el presente estudio un Alpha de Cronbach de 0.72 . 
Previo a la aplicación de los cuestionarios se obtuvo la aprobación del Comité de Ética para la investigación con número de registro FAEN-D-1460; y de investigación de la Facultad de Enfermería de la Universidad Autónoma de Nuevo León; asimismo se contó con la autorización de las instituciones sociales. Para ello se presentó con cada uno de los responsables de las instituciones y se les explicó el objetivo del estudio, procedimiento e instrumentos de medición, además se solicitó un área específica para realizar las entrevistas.

Posteriormente se realizó la invitación a participar a los AM, se explicó el propósito de la investigación, el tiempo de duración de la entrevista, se resaltó la confidencialidad y el anonimato, se evaluó que el AM estuviera orientado en tiempo, espacio y persona y la presencia de deterioro cognitivo. Una vez que el AM aceptó colaborar se procedió a la entrevista que inició con la lectura y firma del consentimiento informado, después se le entregó una copia del consentimiento. Una vez que el AM autorizó participar se le entregaron copias de los instrumentos para que pudiera leer las preguntas y opciones de respuesta en caso de no recordarlas; al finalizar la recolección de datos se le agradeció por su participación y se le acompaño al salón principal para que continuara con sus actividades.

El estudio se apegó a lo establecido por el Reglamento de la Ley General de Salud en Materia de Investigación para la Salud ${ }^{29}$ en México. De igual forma, se contó con el consentimiento informado con el fin de obtener la aceptación y autorización del AM para participar en el estudio, en el cual se garantizó el anonimato y la confidencialidad de la información proporcionada por este.

Los datos fueron analizados a través del programa estadístico SPSS versión 21 para Windows. Se utilizó estadística descriptiva e inferencial, además se utilizó la prueba de normalidad de Kolmogorov-Smirnov con Correccion de Lilliefors y de acuerdo con el resultado se decidió el uso de estadística no paramétrica. Finalmente, para dar respuesta al objetivo principal del estudio se empleó el Coeficiente de Correlación de Spearman y el Modelo de Regresión Lineal Simple.

\section{RESULTADOS}

77.8\% de los AM pertenece al sexo femenino, la media de edad fue de 69.5 años ( $\mathrm{DE}=6.9$ ), con relación al estado civil $60.2 \%$ reportó estar casado, $25 \%$ viudo, $11.1 \%$ divorciado y $3.7 \%$ soltero. En cuanto a la escolaridad, la media de los años de estudio fue de 6.6 años ( $\mathrm{DE}=4.0$ ), por ello $45.4 \%$ tenía estudios básicos de primaria, 17.6\% secundaria, 21.3\% estudios técnicos y solo $2.8 \%$ preparatoria, $3.7 \%$ licenciatura, $9 \%$ posgrado y $8.3 \%$ sin estudios. Con respecto a la ocupación, $59.3 \%$ se dedicaba al hogar, $25.9 \%$ eran jubilados y $6.5 \%$ trabajaba en un comercio menor, $7.4 \%$ contaba con un negocio propio y $0.9 \%$ era cuidador de algún familiar. En lo referente a la religión, 95.4\% se identificó como católico, $2.8 \%$ cristiano y $1.9 \%$ ateo.

En relación con las variables de interés, en la Tabla 1 se observa que el índice general del SOC mostró una media de 70.1 puntos ( $\mathrm{DE}=12.1$ ), lo cual indica un alto SOC en los $\mathrm{AM}$; en cuanto a los EEV se observó una media de 15.1 puntos ( $\mathrm{DE}=13.6$ ), por lo que se observa que los $\mathrm{AM}$ han experimentado pocos EEV en los últimos doce meses.

\begin{tabular}{lcccccc} 
& \multicolumn{6}{c}{ Tabla 1. Medidas de tendencia central de variables de interés } \\
Variable & Media & Mdn & DE & Rango & Mínimo & Máximo \\
\hline SOC* & 70.1 & 71.0 & 12.1 & 66 & 23 & 89 \\
\hline EEV ${ }^{-1}$ & 15.1 & 11.5 & 13.6 & 71 & 0 & 71 \\
\hline
\end{tabular}

Nota: $\mathrm{SOC}=$ Sentido de Coherencia, $† \mathrm{EEV}=$ Evento Estresante de la Vida, $\neq \mathrm{DE}=$ Desviación estándar 
Respecto a los EEV en la Tabla 2 se muestran aquellos que fueron más experimentados por los $\mathrm{AM}$ en los últimos doce meses, entre los que destacan: el deterioro de la memoria (54.6\%), la presencia de una lesión o enfermedad (50.0\%), la alteración en la salud de algún miembro cercano a la familia (48.1\%), el no poder realizar actividades que disfrutaba mucho (45.5\%) y el deterioro del estado financiero (37.0\%).

\begin{tabular}{lc}
\multicolumn{2}{c}{ Tabla 2. EEV más experimentados en los últimos doce meses } \\
Eventos & $\%$ \\
El deterioro de la memoria & 54.6 \\
\hline La presencia de una lesión o enfermedad en el AM & 50.0 \\
\hline La alteración en la salud de algún miembro de la familia & 48.1 \\
\hline No poder realizar actividades que disfrutaba mucho & 45.5 \\
\hline El deterioro del estado financiero & 37.0 \\
\hline La muerte de un miembro cercano a la familia & 35.2 \\
\hline El deterioro en las condiciones de vida & 31.5 \\
\hline La muerte de un amigo cercano & 30.6 \\
\hline Problemas de comunicación y relación con algún hijo(a) & 28.7 \\
\hline \multicolumn{2}{c}{ Nota: EEV = Evento Estresante de la Vida } \\
\hline
\end{tabular}

Para dar respuesta al objetivo principal del estudio se utilizó primero el Coeficiente de Correlación de Spearman, se encontró una relación negativa y significativa entre el SOC y los EEV $(r s=-.557, p<.01)$, es decir, a mayor SOC, menor percepción de estrés en los AM. Después se realizó el análisis de correlación por cada una de las dimensiones del SOC con los EEV, se observó una relación negativa y significativa entre las tres dimensiones del SOC; 1 ) significatividad ( $r s=-.427, p<.01), 2$ ) manejabilidad ( $r s=-.495, p<.01)$ y 3 ) comprensibilidad $(r s=-.414, p<.01)$ con los EEV, siendo la dimensión de manejabilidad la que tuvo una relación más fuerte, por lo que a mayor significatividad, manejabilidad y comprensibilidad, menor percepción de estrés en los AM.

Con el fin de conocer el efecto del SOC en los EEV se empleó el Modelo de Regresión Lineal Simple como se muestra en la Tabla 3, el cual explicó $42 \%$ del total de la varianza, por ello el SOC fue predictor negativo y significativo de los EEV ( $\beta=-.652, p=.001$ ). En consecuencia, los altos puntajes de SOC darían lugar a una menor percepción de estrés ante los eventos de la vida experimentados en los últimos doce meses en los AM.

\begin{tabular}{lcccc}
\multicolumn{5}{c}{ Tabla 3. Modelo de Regresión Simple SOC y EEV } \\
Variable & B & $\beta$ & $\boldsymbol{t}$ & $\boldsymbol{p}$ \\
SOC & -.729 & -.652 & -8.8 & .001 \\
\hline \multicolumn{5}{c}{ Nota: Variable independiente SOC }
\end{tabular}

\section{DISCUSIÓN}

El perfil sociodemográfico de los participantes mostró que en su mayoría pertenecen al sexo femenino, la escolaridad fue de nivel primaria y más de la mitad reportó estar casado. Estas características 
son comunes en la población de AM en México debido a que la esperanza de vida para las mujeres es mayor (78 años) en comparación con los hombres (73 años)3o. En cuanto al nivel educativo, el promedio de los años de estudio de los AM es de 4.6, el cual tiende a ser bajo ya que esta generación tuvo menores oportunidades de estudio y una alta necesidad de trabajar desde temprana edad ${ }^{3}$.

Respecto a la ocupación, más de la mitad de los AM se dedica al hogar, menos del 10\% continúa trabajando, pero en el mercado informal; 73.9\% de los hombres y $12.9 \%$ de las mujeres contaban con una pensión limitada dado el tipo de trabajo que desempeñaron. En México sólo 34.1\% de los AM pertenecen a la población económicamente activa y existen diferencias marcadas entre mujeres (30\%) y hombres (70\%); además sólo 35\% de los AM cuentan con una pensión, de los cuales 39\% son hombres y $31 \%$ son mujeres. Esto último se explica porque en la etapa adulta trabajaron en su mayoría en el mercado informal que no da derecho a un retiro y por ende no les permite tener acceso a la seguridad social ${ }^{3-32}$.

El índice general del SOC mostró altos puntajes, similar a lo reportado por Andruszkiewicz et al. ${ }^{14}$, Potier et al..$^{18} \mathrm{y}$ Takahashi et al. ${ }^{20}$; en relación con los EEV el índice general mostró puntajes bajos, lo cual concuerda con otro estudio realizado por Munro et al. ${ }^{33}$ en AM. Estos resultados pueden explicarse a través de la visión salutogénica desarrollada por Antonovsky que indica que las situaciones difíciles experimentadas en el pasado por el individuo pueden ser afrontadas cuando el SOC es alto, también este puede incrementarse a lo largo de la vida y aumentar la capacidad de comprender, manejar y dar significado a dichos eventos, aun aquellos que suelen ser potencialmente estresantes ${ }^{10,16,34}$.

En relación con los EEV experimentados en los últimos 12 meses por los AM, destacaron el deterioro de la memoria, la presencia de una lesión o enfermedad en el AM, la alteración en la salud o en la conducta de un familiar, el no poder realizar actividades y el deterioro del estado financiero, mismos que pueden ser considerados como pérdidas; esto es similar a los estudios realizados por Rubio et $a l .{ }^{10}$, Lim et al..$^{35}$ y Zorigt et $\mathrm{al}^{36}$. La vejez es considerada como una etapa en la que se producen numerosos cambios que se asocian con pérdidas significativas de la vida, como la pérdida de la independencia, del estado de salud y el estatus socioeconómico. Lo que es más, si los AM no cuentan con el apoyo social de personas significativas, no logran adaptarse satisfactoriamente a estos eventos que se convierten en una fuente de estrés ${ }^{37-38}$.

En cuanto al objetivo principal del estudio, los resultados mostraron que el SOC tuvo relación y efecto negativo y significativo con los EEV, esto concuerda con las investigaciones realizadas en AM por Larm et al. ${ }^{19}$, Andruszkiewic et al. ${ }^{14}$, Potier et al. ${ }^{18}$, Guo et al. ${ }^{21}$, Sandoval-Carlos et al. ${ }^{37}$ y Stensletten et $\mathrm{l}^{39}$. Lo anterior puede deberse probablemente a las experiencias de vida del AM, las cuales le han permitido desarrollar un alto grado de SOC que lo ha capacitado para percibir y seleccionar los recursos que tiene a su disposición para hacer frente a los EEV y de este modo disminuir sus efectos negativos, considerar el mundo y su entorno inmediato como menos amenazante, mantener una actitud positiva y un alto grado de salud ${ }^{40-44}$.

La dimensión de "manejabilidad" mostró una relación más fuerte con los EEV, similar a lo reportado por López et al44. Esto puede explicarse en razón de que los AM del presente estudio asistían a algunas instituciones sociales, las cuales en México les brindan un espacio para su recreación y algunos servicios básicos como los de salud, asistencia jurídica y orientación social, socialización, educación y capacitación, por lo cual pueden contribuir a aumentar el SOC. Cabe señalar que este último les ha permitido identificar y aprovechar estos recursos para hacer frente a los EEV41-43.45. 


\section{CONCLUSIONES}

Los resultados mostraron que la mayoría de los participantes pertenecía al sexo femenino, tenía estudios básicos de primaria y se dedicaba al hogar y sólo una pequeña proporción contaba con una pensión o jubilación. En cuanto a las variables de interés se observó una media alta en el índice general del SOC y una media baja en el índice general de los EEV en los AM. Entre los EEV más experimentados por los AM, destacan las pérdidas como el deterioro de las capacidades cognitivas, la disminución del estado de salud o la presencia de enfermedad de carácter importante, la pérdida del estado financiero, pérdida de las condiciones de vida, el no poder realizar actividades que disfrutaba mucho y la muerte de seres queridos como un hijo o esposo. Respecto al objetivo principal del estudio se encontró una relación y efecto negativo y significativo entre el SOC y los EEV; asimismo, la dimensión de manejabilidad mostró una relación más fuerte con los EEV.

Los resultados de este trabajo pueden ser de utilidad para que en un futuro se generen nuevas investigaciones con muestras más grandes y en otros contextos como la comunidad abierta. De igual forma, los resultados de este estudio podrían ser una fuente de información para que el personal del área de la salud realice acciones que fortalezcan las capacidades y habilidades de los AM, con el fin de identificar y seleccionar los recursos internos y externos a su disposición para hacer frente a los eventos de vida potencialmente estresantes, de esta manera se coadyuva al bienestar y la calidad de vida.

Este fenómeno es de vital importancia ya que el AM está constantemente expuesto a situaciones de la vida estresantes y al no identificar o no contar con los recursos internos o externos, puede emplear prácticas no saludables para hacer frente a dichos EEV, como el consumo de drogas médicas o de alcohol. Entre las limitaciones del estudio se encuentran el no haberlo realizado en población abierta debido a la inseguridad que existe en las comunidades y que puede afectar a los investigadores del estudio.

\section{RESPONSABILIDADES ÉTICAS}

Protección de personas o animales. En este estudio no se realizaron experimentos en personas o animales.

Confidencialidad. Se obtuvo el consentimiento informado, además se garantizó el anonimato y la confidencialidad de la información proporcionada.

Conflicto de intereses. Los autores señalan que no existe conflicto de interés.

Financiamiento. Ninguno.

\section{REFERENCIAS}

1. Organización Mundial de la Salud. Envejecimiento y salud. Ginebra: OMS; 2018. https://bit.ly/zfuU8Lo

2. Organización Mundial de la Salud. 10 datos sobre el envejecimiento y la salud. Ginebra: OMS; 2017. https://bit.ly/39b7FH5

3. Instituto Nacional de Estadística y Geografía. Estadísticas a propósito del día internacional de las personas de edad ( $1^{\circ}$ de octubre) datos nacionales. México: INEGI; 2018. https://bit.ly/2IVzFnt

4. Consejo Nacional Para Prevenir la Discriminación. Ficha temática. Personas mayores. México: CONAPRED; 2017.

5. Dirección General de Comunicación Social. Adultos mayores en México enfrentan pobreza, violencia y atención con modelos obsoletos. México: UNAM-DGCS; 2018. https://bit.ly/3m28Xb5 
6. Instituto Nacional de Estadística y Geografía. Encuesta Nacional de la Dinámica Demográfica (ENADID). 2018. México: INEGI; 2018. https://bit.ly/3m4H16d

7. Holmes TH, Rahe RH. The social readjustment rating scale. J Psychosom Res. 1967; 11(2): 213-8. https://doi.org/10.1016/0022-3999(67)90010-4

8. Aldwin CM. Stress, coping, and debelopment. An integrative perspective. 2nd ed. New York: Guilford Press; 2007 .

9. Chukwuorji JC, Nwoke MB, Ebere MO. Stressful life events, family support and successful ageing in the Biafran War generation. Aging Ment Health. 2017; 21(1), 95-103. https://doi.org/10.1080/13607863.2015.1083946

10. Rubio L, Dumitrache C, Cordon-Pozo E, Rubio-Herrera R. Coping: Impact of gender and stressful life events in middle and in old age. Clin Gerontol. 2016; 39(5): 468-88.

https://doi.org/10.1080/07317115.2015.1132290

11. Friborg O. An improved method for counting stressful life events (SLEs) when predicting mental health and wellness. Psychol Health. 2019; 34(1): 64-83.

https://doi.org/10.1080/08870446.2018.1516768

12. Cleland C, Kearns A, Tannahill C, Ellaway A. Home truths: Are housing-related events more important for residents' health compared with other life events? Hous Stud. 2016; 31(5): 495-518.

https://doi.org/10.1080/02673037.2015.1094565

13. Monma T, Takeda F, Okura T. Physical activities impact sense of coherence among communitydwelling older adults. Geriatr Gerontol Int. 2017; 17(11): 2208-15. https://doi.org/10.1111/ggi.13063

14. Andruszkiewicz A, Basińska MA, Felsmann M, Banaszkiewicz M, Marzec A, Kędziora-Kornatowska $\mathrm{K}$. The determinants of coping with pain in chronically ill geriatric patients - the role of a sense of coherence. Clin Interv Aging. 2017; 12: 315-23. https://doi.org/10.2147/CIA.S118136

15. Sarenmalm EK, Browall M, Persson LO, Fall-Dickson J, Gaston-Johansson F. Relationship of sense of coherence to stressful events, coping strategies, health status, and quality of life in women with breast cancer. Psychooncology. 2013; 22(1): 20-7. https://doi.org/10.1002/pon.2053

16. Forbech-Vinje H, Langeland E, Bull T. Aaron Antonovsky's development of salutogenesis. En: Mittelmark MB, Sagy S, Eriksson M, Bauer G, Pelikan JM, Lindström B, et al. (Eds). The handbook of salutogenesis. Switzerland: Springer; 2017. https://doi.org/10.1007/978-3-319-04600-6

17. Da Rosa-Possebon AP, Pinto-Martins AP, Freire-Danigno J, De Oliveira-Langlois C, Ribeiro-Silva AE. Sense of coherence and oral health in older adults in Southern Brazil. Gerodontology. 2017; 34(3): 377-81. https://doi.org/10.1111/ger.12276

18. Potier F, Degryse JM, Henrard S, Aubouy G, de Saint-Hubert M. A high sense of coherence protects from the burden of caregiving in older spousal caregivers. Arch Gerontol Geriatr. 2018; 75: 76-82. https://doi.org/10.1016/j.archger.2017.11.013

19. Larm P, Aslund C, Starrin B, Nilsson KW. How are social capital and sense of coherence associated with hazardous alcohol use? Findings from a large population-based Swedish sample of adults. Scand J Public Health. 2016; 44(5): 525-33. https://doi.org/10.1177/1403494816645221

20. Takahashi K, Kato A, Igari T, Sase E, Shibanuma A, Kikuchi K, et al. Sense of coherence as a key to improve homebound status among older adults with urinary incontinence. Geriatr Gerontol Int. 2015; 15(7): 910-7. https://doi.org/10.1111/ggi.12353

21. Guo LN, Liu YJ, McCallum J, Söderhamn U, Ding XF, Yv SY, et al. Perceived stress and depression amongst older stroke patients: Sense of coherence as a mediator? Arch Gerontol Geriatr. 2018; 79: 164-70. https://doi.org/10.1016/j.archger.2018.08.010 
22. Peña-Méndez A. $4^{\circ}$ Encuentro de Jóvenes Investigadores - CONACYT $11^{\circ}$ Coloquio de Jóvenes Talentos en la Investigación. Sentido de coherencia y resiliencia en adultos mayores en el municipio de Villahermosa, Tabasco. Tlamati sabiduría. 2016; 7(2): 1-9.

23. Ugarte-Esquivel A, Serrano-Parra MD, Morales-Jinez A, Trujillo-León YI, López-Rincón FJ, RodríguezMejía LE, et al. Asociación entre sentido de coherencia y nivel de Depresión en los adultos mayores del Norte de México. En: Instituto de Salud Carlos III. Libro de Ponencias. XXI Encuentro Internacional de Investigación en Cuidados. Madrid: Instituto de Salud Carlos III; 2017.

24. Martínez-de la Iglesia J, Dueñas-Herrero R, Onís-Vilches MC, Aguado-Taberné C, Albert-Colomer C, Luque-Luque R. Adaptación y validación al castellano del cuestionario de Pfeiffer (SPMSQ̂) para detectar la existencia de deterioro cognitivo en personas mayores de 65 años. Med. clín. 2001; 117(4);129-34. https://cutt.ly/lnZVr2a

25. Antonovsky H, Sagy S. The development of a sense of coherence and its impact on responses to stress situations. J Soc Psychol. 1986; 126(2), 213-26.

26. Virués-Ortega J, Martínez-Martín P, del Barrio JL, Lozano LM. Validación transcultural de la Escala de Sentido de Coherencia de Antonovsky (OLQ-13) en ancianos mayores de 70 años. Med. clín. 2007; 128(13): 486-92. https://doi.org/10.1157/13100935

27. Alonso-Castillo BA. Situaciones de la vida estresantes, uso y abuso de alcohol y drogas médicas en los adultos mayores de Monterrey, México. Rev. Latino-Am. Enfermagem. 2008; 16(n. spe): 509-15. http://dx.doi.org/10.1590/S0104-11692008000700002

28. Brislin R. The wording and translation of research instruments. En: Lonner WJ, Berry JW. Field methods in cross-cultural research. Newbury Park, California: SAGE Publications; 1986.

29. Secretaria de Salud. Reglamento de la Ley General de Salud en Materia de Investigación para la Salud. México: SSA; 2014. https://bit.ly/362BkjN

30. Instituto Nacional de Estadística y Geografía. Esperanza de vida. México: INEGI; 2016. https://bit.ly/2VcdITH

31. Instituto Nacional de Estadística y Geografía. "Estadísticas a propósito del día internacional de las personas de edad (adultos mayores)" datos nacionales. México:INEGI; 2018. https://bit.ly/3l2xons

32. Instituto Nacional de Estadística y Geografía. Encuesta Nacional de Empleo y Seguridad Social. México: INEGI; 2017. https://bit.ly/3758deW

33. Munro CA, Wennberg AM, Bienko N, Eaton WW, Lyketsos CG, Spira AP. Stressful life events and cognitive decline: Sex differences in the Baltimore Epidemiologic Catchment Area Follow-Up Study. Int J Geriatr Psychiatry. 2019; 34(7): 1008-17. https://doi.org/10.1002/gps.5102

34. Giglio RE, Rodriguez-Blazquez C, de Pedro-Cuesta J, João-Forjaz M. Sense of coherence and health of community-dwelling older adults in Spain. Int Psychogeriatr. 2015; 27(4): 621-8.

https://doi.org/10.1017/S1041610214002440

35. Lim ML, Lim D, Gwee X, Nyunt MSZ, Kumar R, Ng TP. Resilience, stressful life events, and depressive symptomatology among older Chinese adults. Aging Ment Health. 2015; 19(11): 1005-14. https://doi.org/10.1080/13607863.2014.995591

36. Zorigt G, Enkh-Amgalan N, Yu T. Use of best-worst scaling to estimate the magnitude of stressful life events in older adults. Psychogeriatrics. 2019; 19(3): 212-8. https://doi.org/10.1111/psyg.12384

37. Sandoval-Carlos KC, García-Sánchez MD, Luis-Delgado OE. Pérdidas a lo largo del ciclo vital en adultos mayores. Psicol. salud. 2019; 29(1): 79-90.

38. Kozlova I, Parra MA, Della-Sala S. Acreemagnosia (loss of financial knowledge): a symptom of functional and cognitive loss in frail elderly. Int J Geriatr Psychiatry. 2018; 33(2): 434-5.

https://doi.org/10.1002/gps.4744 
39. Stensletten K, Bruvik F, Espehaug B, Drageset J. Burden of care, social support, and sense of coherence in elderly caregivers living with individuals with symptoms of dementia. Dementia. 2016; 15(6): 1422-35. https://doi.org/10.1177/1471301214563319

40. Del-Pino-Casado R, Espinosa-Medina A, López-Martínez C, Orgeta V. Sense of coherence, burden and mental health in caregiving: A systematic review and meta-analysis. J Affect Disord. 2019; 242: 14-21. https://doi.org/10.1016/j.jad.2018.08.002

41. Nilsen C, Andel R, Fritzell J, Kareholt I. Work-related stress in midlife and all-cause mortality: can sense of coherence modify this association? Eur J Public Health. 2016; 26(6): 1055-61.

https://doi.org/10.1093/eurpub/ckwo86

42. Arola LA, Barenfeld E, Dahlin-Ivanoff S, Häggblom-Kronlöf G. Distribution and evaluation of sense of coherence among older immigrants before and after a health promotion intervention - results from the RCT study promoting aging migrants' capability. Clin Interv Aging. 2018; 13: 2317-28. https://doi.org/10.2147/CIA.S177791

43. Jueng RN, Tsai DC, Chen IJ. Sense of coherence among older adult residents of long-term care facilities in Taiwan: A cross-sectional analysis. PloS one. 2016; 11(1): 1-12.

https://doi.org/10.1371/journal.pone.0146912

44. López J, Camilli C, Noriega C. Posttraumatic growth in widowed and non-widowed older adults: Religiosity and sense of coherence. J Relig Health. 2015; 54(5): 1612-28.

https://doi.org/10.1007/s10943-014-9876-5

Instituto Nacional de Desarrollo Social. Ley de Asistencia Social. México: Gobierno de México; 2018. https://ng.cl/zquh 